\title{
g-3. Measurement of Cerebral Blood Flow under Hypothermia
}

\author{
Shigemasa IKeda, Toshiki Chikamitsu, Yasuhiro FuJII, Toshiki AJI \\ and Futami KosaKa \\ Department of Anesthesiology, Okayama University Medical School
}

A few investigations on the cerebral blood flow under hypothermia were previously reported. Most of them were investigated by the so-called KetySchmidt's nitrous oxide method or its modified methods.

The solubility of gases increases at lower temperature. Therefore, a special consideration is required when the cerebral blood flow under hypothermia is calculated by the nitrous oxide method.

Authors previously reported that partition ratios (cerebral tissue/cerebral venous blood) of nitrous oxide under hypothermia are variable figures influenced by body temperature and inhalation time, and that, for the appropriate application of nitrous oxide method for measurement of cerebral blood flow under hypothermia, these varied values should be used for partition coefficient, instead of 1.0 reported by Kety et al.

In our experiments on dogs, direct measurement of cerebral hemodynamics during hypothermia were performed by means of two pairs of electromagnetic flowmeters attached to the bilateral common and external carotid arteries.

Experimental animals were surface-cooled, artificially ventilated, the arterial $\mathrm{pCO}_{2}$ maintained from 20 to $25 \mathrm{mmHg}$ during the experiment. The arterial blood pressure was monitored continuously by cannulation of the femoral artery coupled to a pressure transducer. Temperatures were recorded from a thermister inserted into the femoral artery.

Cerebral blood flow during hypothermia decreased progressively and proportionately with changes in temperature. Cerebral blood flow was reduced to approximately $30 \%$ of control value at $30^{\circ} \mathrm{C}$, while the arterial blood pressure decreased to about $70 \%$ of the control value. It shows that the arterial blood pressure is not a good manifestation of the cerebral blood flow under hypothermia.

These results in our experiments were also compared with those obtained by other investigators. The values available from the literatures were corrected by a partition ratios proposed by us. The corrected values corresponded well to those obtained in our experiments. 